\title{
Studying Collective Human Decision Making and Creativity with Evolutionary Computation
}

\author{
Hiroki Sayama* $\quad$ Shelley D. Dionne ${ }^{\dagger}$
}

\begin{abstract}
We report a summary of our interdisciplinary research project "Evolutionary Perspective on Collective Decision Making" that was conducted through close collaboration between computational, organizational and social scientists at Binghamton University. We redefined collective human decision making and creativity as evolution of ecologies of ideas, where populations of ideas evolve via continual applications of evolutionary operators such as reproduction, recombination, mutation, selection, and migration of ideas, each conducted by participating humans. Based on this evolutionary perspective, we generated hypotheses about collective human decision making using agent-based computer simulations. The hypotheses were then tested through several experiments with real human subjects. Throughout this project, we utilized evolutionary computation (EC) in non-traditional ways-(1) as a theoretical framework for reinterpreting the dynamics of idea generation and selection, (2) as a computational simulation model of collective human decision making processes, and (3) as a research tool for collecting high-resolution experimental data of actual collaborative design and decision making from human subjects. We believe our work demonstrates untapped potential of EC for interdisciplinary research involving human and social dynamics. 1
\end{abstract}

Keywords: Evolution of ideas, collective human decision making, collaborative design, creativity, agent-based simulation, human subject experiments, evolutionary computation, hyperinteractive evolutionary computation.

${ }^{*}$ Collective Dynamics of Complex Systems Research Group / Departments of Bioengineering \& Systems Science and Industrial Engineering, Binghamton University, Binghamton, New York 13902-6000. sayama@ binghamton.edu

${ }^{\dagger}$ Center for Leadership Studies, School of Management, Binghamton University, Binghamton, New York 13902-6000. sdionne@binghamton.edu

${ }^{1}$ This paper is an extended version of [1]. 


\section{Introduction}

In Artificial Life and other computational science and engineering research areas, evolutionary computation (EC) has been widely used as metaheuristics for nonlinear search, design and optimization [2, 3]. From a different point of view, however, EC also has great potential as a theoretical model that operationalizes complex evolutionary processes in a simple, tractable formalism. One application area of EC as a modeling tool is collective human decision making and creativity [4, 5, 6], which typically involves high-dimensional nonlinear problem space, nontrivial societal structure, within-individual cognitive and behavioral patterns, and/or between-individual diversity.

In our project "Evolutionary Perspective on Collective Decision Making" [7], we used EC as a concise, yet sufficiently comprehensive, theoretical/computational model of the dynamics of collective human decision making. We proposed a fundamental shift of the viewpoint from the properties and behaviors of human participants (as in most existing literature) to the properties and behaviors of ideas being discussed. Collective decision making and creative processes are redefined as evolution of ecologies of ideas over a social network habitat, where populations of ideas evolve via continual applications of evolutionary operators such as reproduction, recombination, mutation, selection, and migration of ideas, each conducted by participating humans. This paper summarizes how we utilized EC in several non-traditional ways in this project.

\section{Theoretical Framework}

The framework we propose views idea generation and selection in collective human decision making as an evolutionary process, by shifting the viewpoint from individual participants' personal properties and decisions to the dynamical evolution of ideas being discussed. Obviously, this is a drastic simplification of real humans' social group dynamics that are very complex, which could involve various interests, motives and strategies that would hugely influence discussion outcomes. Here, we limit ourselves to studying how much we could model, understand and predict group decision making by focusing on temporal changes of ideas and describing them as evolutionary processes.

In our framework, several key concepts developed and used in evolutionary theory can be mapped to collective decision making as in Table 1. Many evolutionary operators are conceivable as a representation of diverse human behaviors, as described below.

For selection-oriented operators, replication of an idea is a form of positive selection, which represents 
Table 1: Mapping of concepts from evolutionary theory to concepts in human decision making (items with asterisks (*) do not exist in real biology, however).

\begin{tabular}{lll}
\hline Category & Concepts in evolution & Concepts in human decision making \\
\hline Genetics & Genetic possibility space & Problem space \\
& Genome & Idea (a set of choices for all aspects of the problem) \\
& Locus on a genome & Aspect of the problem \\
& Allele & Specific choice made for an aspect \\
\hline Ecology & Population & A set of ideas being discussed \\
& Environment & A group of individual participants and their utility functions \\
& Fitness & Utility value of an idea (either perceived or real) \\
& Adaptation & Increase of utility values achieved by the idea population \\
\hline Evolutionary & Replication & Advocacy (increase of relative popularity of an idea) \\
Operator & Mutation & Minor modification of an idea \\
& Recombination & Production of a new idea by crossing multiple ideas \\
& Subtractive selection & Criticism (narrowing of diversity of ideas based on their fitness) \\
& Intelligent point mutation* & Improvement of an existing idea \\
& Random generation* & "Crazy" inspiration out of nowhere \\
\hline
\end{tabular}

advocacy of a particular idea under discussion. Similarly, criticism against an idea may be considered a form of negative, subtractive selection that eliminates ideas with poor utility from the population. Subtractive selection serves to reduce the number of ideas under consideration. Both positive and negative selections seek to narrow decision possibilities based on "fitness" among decision makers, in the expectation that it will increase the overall fitness of ideas being discussed.

For variation-oriented operators, random point mutation adds a copy of an idea with point mutations, making random changes to existing ideas by asking "what if"-type questions. Likewise, intelligent point mutation initially begins like random point mutation, however several variations from the original idea may be tried internally within a human individual, and then the idea with the highest perceived fitness will be selected and proposed. Intelligent point mutation is not present in biological evolution but still is relevant to our framework, because it represents hill climbing nature of human thinking. Recombination and random generation also may be considered within variation. Recombination represents the creation of a new idea by crossing two (or more) existing ideas. Random generation represents a sudden inspiration of a completely novel idea unrelated to the existing ideas (which may arise entirely randomly or by drawing on aspects of the 
individual's personal experiences outside the bounds of the discussion). These variation operations enhance the exploratory capabilities of the population, but generally reduce their immediate fitness.

Here we note that the specific choices of evolutionary operators presented in this paper are neither a focus nor a main contribution of our work. Rather, our emphasis is on the significance of the proposed modeling framework itself, with which one can develop many different evolutionary operators based on the human behavioral patterns of interest and easily implement them in simulations.

We are not the first to consider idea dynamics instead of participant dynamics in collective decision making. For example, classic research on the Delphi methodology [8] investigated the dynamics of idea convergence through standardized voting mechanisms that experts can use as a tool to assess ideas and decisions. More relevant to our framework is group decision support research via computer mediated communication (CMC) [9, 10, 11]. Evolutionary theory, in contrast, will offer a more generic yet mechanistic framework to model the whole processes from idea generation to decision selection, by which one can understand and produce hypotheses about how the specifics of decision frameworks, such as bias, team structure, and homogeneity/heterogeneity of individual and group decision criteria, may impact decision processes and outcomes. Our framework is also related to the concept of memes [12] and memetic evolutionary studies [13] that attempt to describe human cultural evolution through the transfer of pieces of cultural information over human populations. Our research is distinct from memetics in that we specially focus on collective decision making that takes place in timescale orders of magnitude shorter than that of human cultural evolution. Moreover, our research aims to produce prescriptive implications for general or specific tendencies of collective decision making dynamics to help improve real-world human decision making problems, which may also contribute to memetics that is so far mostly descriptive in nature.

\section{Computer Simulation Model}

We applied the evolutionary framework introduced above to develop a computer simulation model of group decision making processes within a small-sized, fully connected social network structure [14]. This is a straightforward application of EC to the evolution of ideas, with additional assumptions made to represent multiple human participants with different worldviews (i.e., fitness evaluation criteria) and different behavioral traits (i.e., relative frequencies of evolutionary operator usage).

Our model consists of $N$ agents that are initiated with a shared population of $k$ randomly generated ideas and then take turns in applying various evolutionary operators to the idea population for $T$ iterations (Fig. 11). 


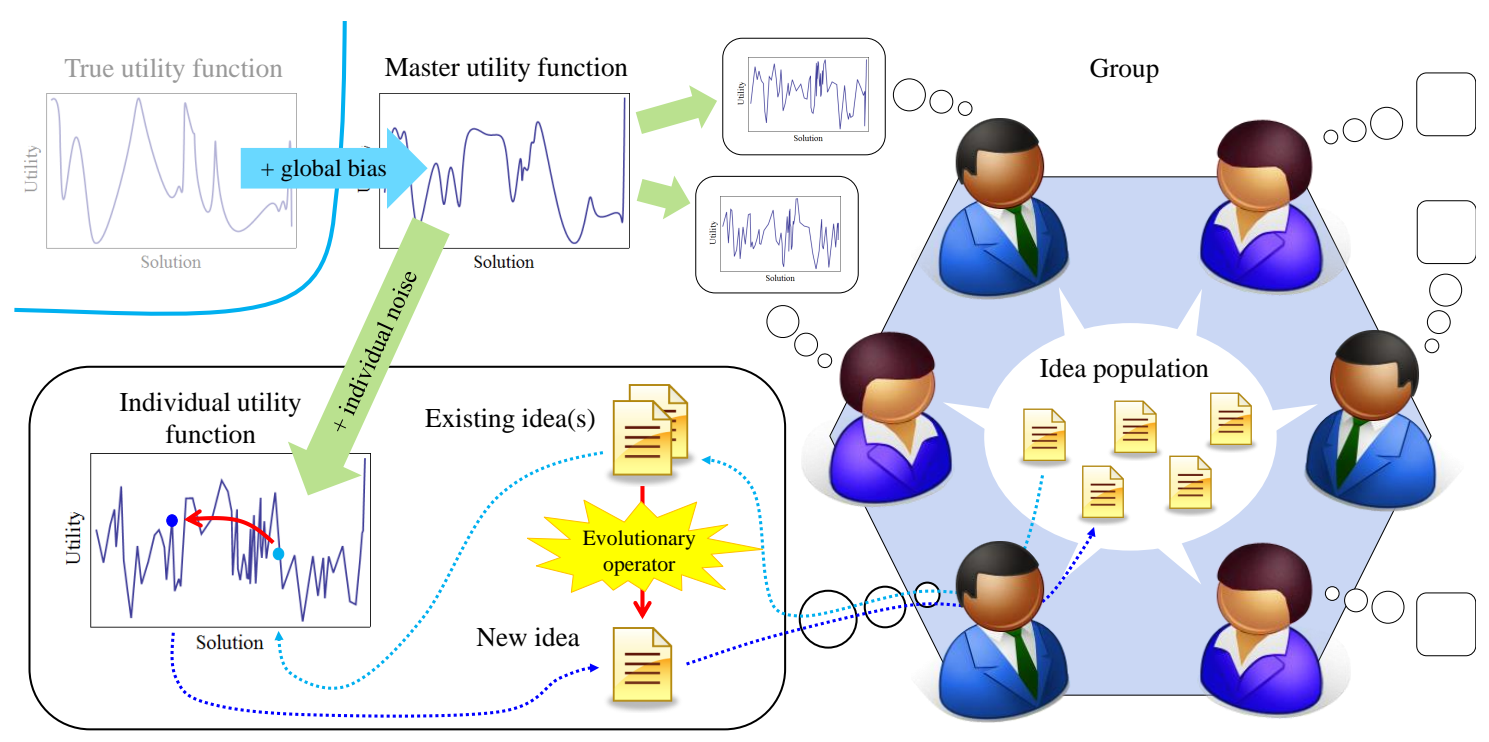

Figure 1: An overview of our computer simulation model of group decision making processes.

Individuals always act in the same order and groups always demonstrate a full rotation. The total number of actions performed on the population in a simulation is thus $N T$. In the population of ideas being discussed, there may exist multiple copies of an identical idea, which represents the relative popularity of that idea among group members. We assumed that actions were performed on single copies, not the equivalence class of all idea replicates.

The group of agents is situated in an $M$-dimensional binary problem space, with $2^{M}$ possible ideas. For a simulation, every idea has a utility value specified by a master utility function $U$ that is unavailable to group members. Individuals perceive idea utility values based on their own utility functions $U_{j}$ constructed by adding noise to $U$. A semi-continuous assignment of utility values is defined over the problem space in the following way. First, $n$ representative ideas $S=\left\{v_{i} \mid i=1 \ldots n\right\}$ are randomly generated, where each $v_{i}$ represents one idea made of $M$ bits. One idea is assigned the maximum fitness value, 1 , and another, the minimum fitness value, 0 , to ensure the range of utility values are always $[0,1]$. The remaining $n-2$ ideas are assigned a random real value between 0 and 1 . The master utility function is defined over its whole domain by interpolation using ideas in $S$ and their utility values, i.e.,

$$
U(v)=\frac{\sum_{i=1}^{n} U\left(v_{i}\right) D\left(v_{i}, v\right)^{-2}}{\sum_{i=1}^{n} D\left(v_{i}, v\right)^{-2}},
$$

where $v \notin S$ is the idea in question, $U\left(v_{i}\right)$ is the utility value of a representative idea $v_{i} \in S$, and $D\left(v_{i}, v\right)$ is the Hamming distance between $v_{i}$ and $v$. By using the weighted average, the master utility function is 
defined to have a moderately "smooth" surface in a high-dimensional problem space. Such smoothness of the utility function gives room for intelligent group decision making to outperform merely random decision making.

Each individual agent in a group will have a different set of utility values for the possible ideas. Individual utility functions $U_{j}(v)(j=1 \ldots N)$ are generated by adding random white noise to the master utility function so that

$$
U_{j}(v) \in[\max (U(v)-\nu, 0), \min (U(v)+\nu, 1)]
$$

for all $v$, where $\nu$ is the parameter that determines the amplitude of noise. An example of such an individual utility function in contrast to the master utility function can be found in Supplemental Materials (Fig. S1). Since the direction of noise added to individual utility function varies stochastically from agent to agent, the parameter $\nu$ represents the amount of within-group heterogeneity. The agents do not have access to others' utility functions.

In addition to individual deviations from the master utility function, we also investigated the effect of group-level bias, i.e., common deviations of the master utility function from the "true" utility function. To simulate this, we introduce a new step in the generation of individual utility functions, in which the master utility function $U$ differs from the true utility function $U_{T}$. Specifically, a bias $\beta$ is imposed on the true utility function both by flipping bits with probability $0.25 \beta$ per bit on representative ideas in $S$, and by adding a random noise in $[-\beta, \beta]$ to utility values of the representative ideas. Their utility values are then renormalized to the range $[0,1]$. The master utility function is generated from these biased representative idea set. Subsequent methods follow as described above. Group-level bias represents fidelity of information at the group level, where $\beta=0$ denotes perfect understanding of the problem (at least as a collective), and complete lack of understanding is asymptotically approached as bias increases. Note that having group-level bias does not influence the dynamics of group discussion processes; it only affects the true utility value of the final group decision.

Regarding the agent behavior in discussion, we identified the following six evolutionary operators available to the agents. Although not an exhaustive list, these six operators reflect common forms of action in evolution (either biological or informational) and can be used as analogy for actions in collective human decision making. Some operators use a preferential search algorithm to stochastically search the idea population, where $r_{p}$ ideas are randomly selected and ranked according to their individual utility values by each individual agent, and then the best or worst idea is selected, depending on the nature of the operator. 
Replication adds an exact copy of an idea selected from the population of ideas back onto the population. The idea is chosen for replication with the above-mentioned preferential search algorithm. This represents an advocacy of a particular idea under discussion.

Random point mutation adds a copy of an idea with point mutations, flipping of bits at each aspect of a problem with a probability $p_{m}$. The idea on which the operator acts is chosen from the population with the preferential search algorithm. This represents an attempt of making random changes to the existing ideas, reflected in asking "what if"-type questions.

Intelligent point mutation selects an idea from the population with a preferential search algorithm, makes several $\left(r_{m}\right)$ offspring of the idea by adding random point mutations, and selects that of the highest individual utility for addition to the population. This represents a proposal of an improved idea derived from existing ideas under discussion.

Recombination chooses one idea at random and one with a preferential search algorithm. It then creates two offspring from the two parent ideas with multiple point crossover: parent ideas are aligned by aspects, for each of which there is a probability $p_{s}$ of switching their contents. Then the better offspring is added back to the population. This represents a creation of a new idea by crossing multiple existing ideas.

Subtractive selection deletes from the population the idea with the worst individual utility identified by the (negative) preferential search algorithm. This represents a criticism against a bad idea.

Random generation adds a randomly generated idea to the population. This represents a sudden inspiration of a completely novel idea that is unrelated to the existing ideas under discussion.

Relative frequencies of the use of those operators are assigned to each agent as its own behavioral tendency, which is one of the experimental parameters being varied. Parameter values used for the simulations (provided in Supplemental Materials; Table S1) were set so as to be reasonable in view of typical real group decision making settings. We tested several minor variations for each parameter value, confirming that the results were not qualitatively different from the ones presented below. As experimental conditions, the within-group heterogeneity $\nu$ and the group-level bias $\beta$ were varied from 0 to 1.2 by increments of 0.2 .

Once a simulation is completed, the following two metrics of group performance are measured on the resulting idea population: True utility of the most supported idea (to characterize the quality of the decision 
made by the group), and entropy remaining in the idea population (to characterize the level of consensus within the group). The latter measurement is based on the classical information theoretic definition

$$
H=-\sum_{i=1}^{l} p\left(x_{i}\right) \log _{2} p\left(x_{i}\right),
$$

where $l$ is the number of different types of ideas in the population, and $p\left(x_{i}\right)$ is the ratio of the number of the $i$-th type of idea to the entire population of ideas. The maximal possible entropy for a group playing a sufficient number of rounds is $M$, which occurs when there are exactly $2^{M}$ distinct ideas, all equally represented. Since the entropy represents how many more bits would be needed to completely specify the single final group decision, it can be assumed that $M-H$ is a quantitative measure that intuitively means the effective number of aspects of the problem on which the group has formed a cohesive opinion. To rescale this to the range between 0 and 1 , we use $(M-H) / M$ as a measurement of the level of convergence of group decision.

Figure 2(a) shows the results of computer simulations, plotting the group performance in a 2-D space using the two metrics described above. It was assumed that each agent randomly chose one of the six evolutionary operators with equal probability in each iteration. The results clearly showed that both within-group heterogeneity and group-level bias had negative effects on the true utility value of the most supported idea. While the group-level bias had no adverse effect on the level of convergence, the within-group heterogeneity reduced the level of convergence significantly. The utility achieved by the most heterogeneous groups dropped to just above 0.5 , meaning that there was no net improvement achieved during the group discussion. This was due to the intra-group conflicts of interest among the group members.

We note that the "balanced" acts of group members assumed in the above experiment may be too ideal as a model of actual group members, because real human groups may have biased behavioral patterns. We therefore ran another set of experiments using the same simulation model with different behavioral patterns assumed for different groups. In forming different group properties, we modeled some operators singularly (e.g., random generation was the only dominant operator within the group), and for other groups we combined two evolutionary operators to reflect increasing complexity of group behavior (e.g., recombination and intelligent point mutation). For the former cases, group members were assumed to choose the designated operator for $95 \%$ of their total actions, with $1 \%$ for each of the other five operators. For the latter combined cases, they were assumed to choose each of the two operators for $48 \%$ of their total actions ( $96 \%$ in total), with $1 \%$ for each of the other four operators. Obviously, there are thousands of possible combinations of operators. For this study, however, we limited our examination to eight group types: replication and sub- 
(a)

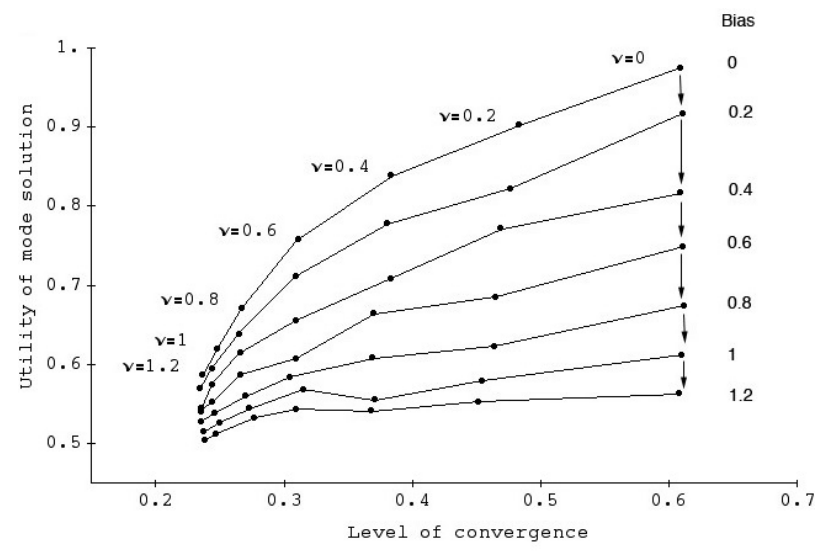

(b)

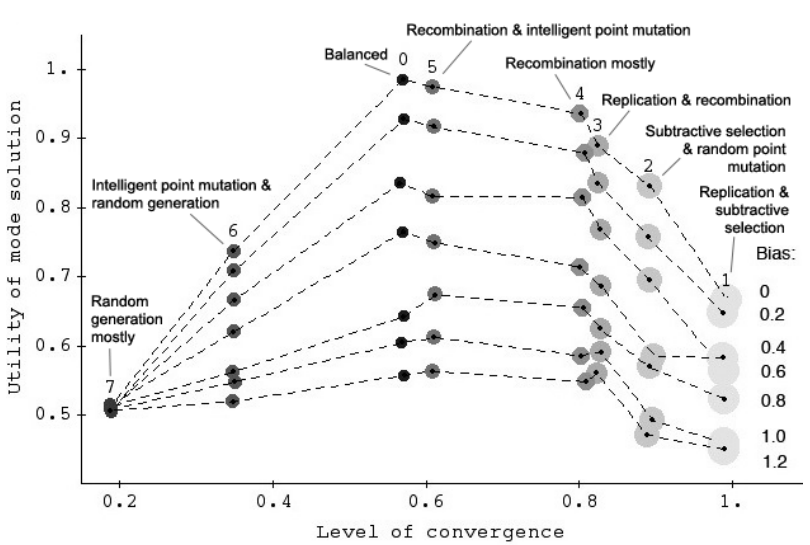

Figure 2: Computer simulation results (horizontal: level of convergence, vertical: true utility value of the most supported idea). (a) Effects of within-group heterogeneity $\nu$ and group-level bias $\beta$ on two outcome metrics. $\nu=0$ represents the case of completely homogeneous groups, while larger values of $\nu$ represent more heterogeneous group cases. Larger values of bias $(\beta)$ represent large discrepancies between the master utility function of the group and the true utility function. (b) Effects of variations in group-level behavioral patterns on two outcome metrics. Within-group heterogeneity $\nu$ was set to 0 . See text for details of the behavioral patterns used in each of the seven different groups.

tractive selection (Group 1); subtractive selection and random point mutation (Group 2); replication and recombination (Group 3); recombination mostly (Group 4); recombination and intelligent point mutation (Group 5); intelligent point mutation and random generation (Group 6); random generation mostly (Group 7); and, finally, the balanced team we used in the previous experiment (Group 0).

Figure 2(b) shows the results. The behaviorally most diverse Group 0 achieved the highest utility value of the most supported idea. Meanwhile, a variety of different group performances were achieved by groups with different balances between selection-oriented and variation-oriented operators, seen as a convergenceutility Pareto frontier near the upper-right corner of the performance space. It is also notable that the random generation operators (used in Groups 6 and 7) were generally harmful to group performance.

We derived from these computer simulations the following three hypotheses:

H1: Within-group homogeneity of problem understanding improves group performance.

H2: Balance between critical and creative attitudes improves group performance. 
H3: Diversity of available evolutionary operators improves group performance.

To test these hypotheses experimentally, we designed and conducted three human subject experiments, which will be described in the following sections. These experiments were mostly conducted in the course 'BE-461: Exploring Social Dynamics' offered to juniors and seniors in the Bioengineering and Management programs at Binghamton University. The study was reviewed and approved by the Binghamton University IRB. No personally identifiable information was collected from the subjects.

\section{Human Subject Experiment 1: Product Name Design}

The first experiment aimed to test Hypothesis H1: Within-group homogeneity of problem understanding improves group performance. The experiments were conducted twice in two separate years (2008 and 2011) using the same protocol described below.

Students were divided into groups of four (or sometimes three). Half of the groups were made of students of the same gender, in the same major, and in the same graduation year, which were intended to represent groups with more homogeneous problem understanding (homogeneous condition). The other half of the groups were made so that the within-group difference of gender, major and year would be as high as possible, which were intended to represent groups with more heterogeneous problem understanding (heterogeneous condition). These groupings were automatically generated by a computer program, but the conditions were hidden from the students. A total of 50 students participated in this experiment, forming 13 groups. The following instruction was given to each group leader:

You are the leader of a marketing team in a trading company that is about to introduce to the U.S. market a new cell phone manufactured by an Asian country. This product has several key features: easy-to-use keypad; easy-to-read display; energy efficiency; long battery life; Bluetooth and WiFi ready. The original name of this product was in foreign language that doesn't sound well to English-speaking people. Your task is to coordinate the team discussion and come up with an attractive name of this product for U.S. customers.

A picture of an ordinary cell phone was also handed to each group to promote their discussion. Once the group reached a consensus, they submitted both their final design (name of the phone) and the whole list of ideas (candidate names) they discussed. Their final designs were then projected to the screen in the classroom and the students ranked the final designs individually and independently. This peer evaluation 
(a)

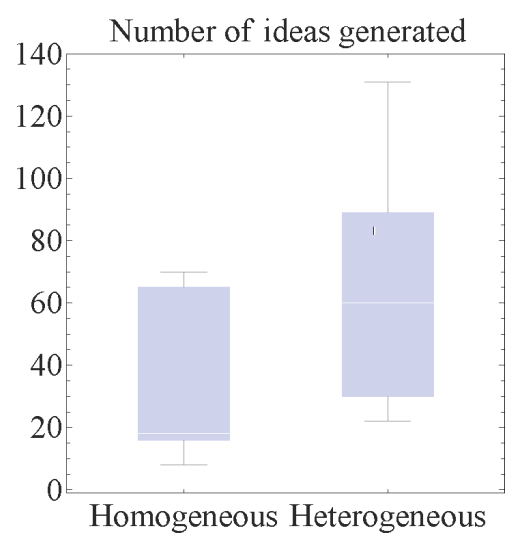

(b)

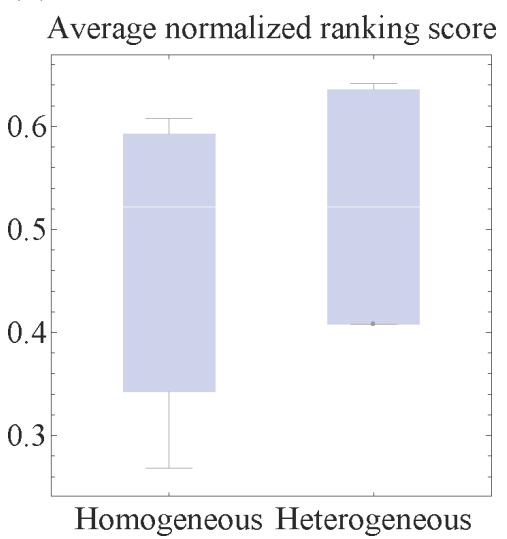

Figure 3: Results of Experiment 1, comparing the distributions of outcomes between homogeneous and heterogeneous groups. (a) Number of ideas generated. A statistically significant difference was detected between the conditions ( $p=0.0456)$. (b) Average normalized ranking score of final designs $(1=$ best, $0=$ worst). No statistically significant difference was detected $(p=0.2816)$.

was used as a measure to quantitatively assess the utilities of the final designs made by each group. The length of the list of all the ideas was also measured as a characteristic of the decision making processes.

The final designs created by the groups under two different conditions are listed in Supplemental Materials (Table S2). Figure 3 compares the numbers of ideas generated and the average ranking scores of the final design between the two conditions. While the sample size was very small, a statistically significant difference was detected in terms of the number of ideas generated (Fig. 3 (3)), i.e., the heterogeneous groups produced more ideas. This can be understood in that the convergence of discussion was relatively easier in homogeneous groups so they did not explore the problem space as much as the heterogeneous groups did. In the meantime, there was no statistically significant difference detected regarding the ranking score of final designs between those two conditions; in fact, groups in the heterogeneous condition appeared to have produced slightly better names (Fig. 3(b)), which may look opposite to what H1 would imply. However, this result actually makes sense when the possible effect of group-level bias is considered. As shown in Fig. 2 (a), the utility of final designs decreases by not only within-group heterogeneity but also group-level bias. It is reasonable to assume that the more diverse the group members are, the better they represent the true utility function (i.e., the preference of the whole class, in this case). Therefore, we interpret this result as a mixture of two different effects of within-group heterogeneity on the quality of final designs - to decrease it due to intra-group conflicts, and to increase it by reducing potential group-level biases. 


\section{Human Subject Experiment 2: Catch Phrase Design}

The second experiment aimed to test Hypothesis H2: Balance between critical and creative attitudes improves group performance. The experiments were conducted twice in two separate years (2008 and 2010) using the same protocol described below.

Students were randomly divided into groups of four (or sometimes three). A total of 45 students participated in this experiment, forming 12 groups. The following instruction was given to each group leader:

You are the leader of a marketing team in a manufacturer of a new laptop computer. The specs of the new laptop are quite mediocre, with no technical feature truly novel and attractive to customers. In any case, your task is to coordinate the team discussion and come up with a list of inspiring catch phrases to promote the sales of the new laptop.

A picture of an ordinary laptop was also handed to each group to promote their discussion. Three different experimental conditions were created by providing the following additional information to selected groups before the discussion:

Critical condition: (4 groups) When coordinating discussion, share the following principles with your team members: Promote and maintain critical attitude throughout the discussion. Always play devil's advocate, trying to find ways for each catch phrase to be potentially problematic. Incremental improvement of existing ideas is the key to making a reliable idea. Completely new ideas will never be better than well-tested ideas.

Creative condition: (4 groups) When coordinating discussion, share the following principles with your team members: Promote and maintain creative attitude throughout the discussion. Always give positive feedback to someone who presented a new idea, trying to find good aspects in it. Crazy inspiration and idiosyncratic thinking is the key to breaking the barrier of stereotyped ideas. Incremental improvement of existing ideas will never work out.

Control condition: (4 groups) No additional instruction was given.

As given in the instruction above, the groups were initially asked to simply produce a list of catch phrases, but after 20 minutes of discussion, they were told to make a final design and choose the best catch phrase out of the produced list. Once the team reached a consensus, they submitted both their final design 
(a)

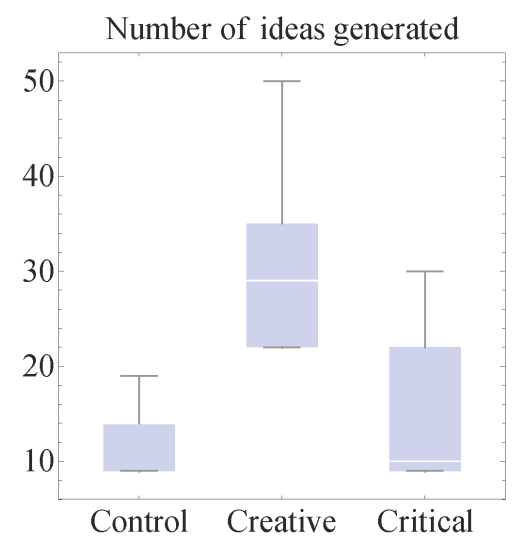

(b)

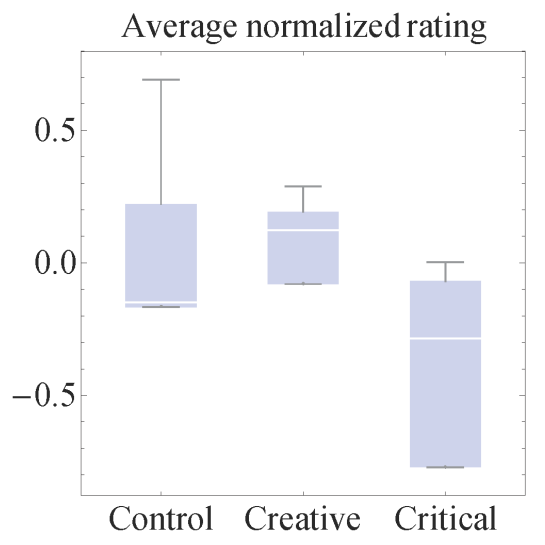

Figure 4: Results of Experiment 2, comparing the mean outcomes between control, creative and critical groups. (a) Number of ideas generated. A statistically significant difference was detected among the three conditions by ANOVA ( $p=0.0314)$, and Tukey's and Bonferroni's posthoc tests showed that there was a significant difference between control and creative conditions. (b) Average normalized rating score of final designs (positive: good, negative: bad). No statistically significant difference was detected by ANOVA $(p=0.156)$.

(catch phrase) and the whole list of ideas (candidate phrases) they discussed. The length of the list of all the ideas was also measured as a characteristic of the decision making processes.

Unlike in the first experiment, the final designs were evaluated by a large number of third parties, i.e., students in another undergraduate course on organizational behavior that had more than 120 enrollments. All the final designs were projected in a randomized order on a screen of a large lecture hall, and then those students in the organizational behavior course rated the quality of each product individually and independently. The average of those rating results was used as the average rating score of each design.

The final designs created by the groups under three different conditions are listed in Supplemental Materials (Table S3). Figure 4 compares the numbers of ideas generated and the average rating scores of the final design between the three conditions. Again, the sample size was quite small for this experiment, but a statistically significant difference was detected among those conditions in terms of the number of ideas generated. Specifically, creative groups produced significantly more ideas than control groups (and seemingly more than critical groups, though with no statistical significance). Also, it was indicated that designs made by critical groups may have received lower ratings than those made by others.

We also transcribed a few sample recordings of the discussions taken in this experiment and manually 
(a) Sample from creative condition

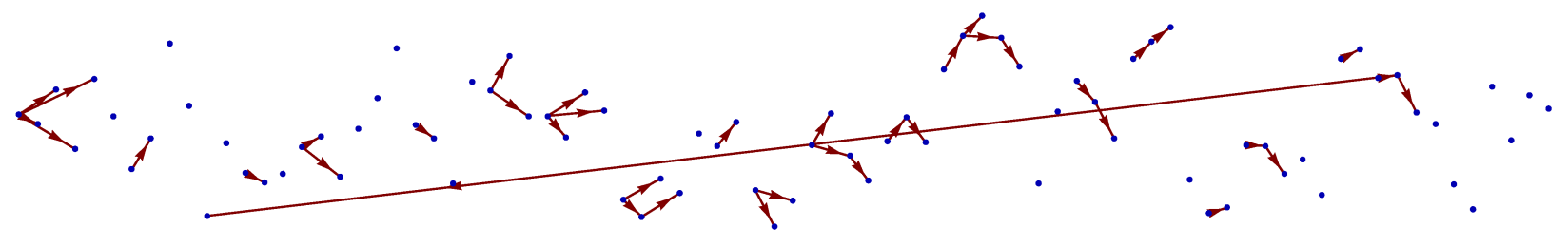

(b) Sample from critical condition

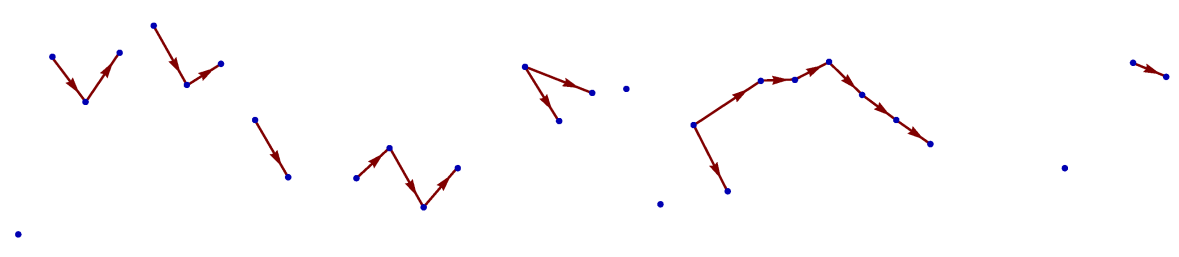

Figure 5: Sample idea genealogies constructed from transcripts of recordings of discussions in Experiment 2. The horizontal axis represents time (not scaled to real time, but arranged in the order of ideas' first appearance in the discussion). The vertical axis was arbitrarily set just for visualization purposes. Each small dot represents a new idea (a catch phrase). An arrow connecting two ideas indicates that a new idea came out of an existing idea, which was detected and noted by human transcribers.

constructed genealogies of ideas that show when new ideas emerged from which existing ideas. Figure 5 shows two illustrative cases, one from a creative group and the other from a critical group. The creative group not only produced more ideas than its critical counterpart, but also showed more "branching" ideation processes to explore various possibilities (Fig. 5(a)). In contrast, the critical group's idea genealogy was mostly sequential with very few branching events (Fig. 5(b)), implying that they produced catch phrases through incremental revisions.

An interesting observation in these results is that the control groups seem to have made collective decisions most efficiently - they conducted as little evolutionary exploration as the critical groups (Fig. 4(a)) and still produced quality solutions comparable to those from the creative groups (Fig. 4(b)). In other words, collective human decision making apparently worked optimally when no additional instruction was given. We interpret these results as follows: Behaviors of people working in groups are most balanced when no explicit instructions are given, leading to best decision outcomes. Instructing teams to be either creative or 
critical may result in loss of behavioral balance and therefore less efficient or less productive discussion, as already seen in our computer simulations (Fig. 2(b)). These findings and observations support Hypothesis $\mathrm{H} 2$, that the balance between critical and creative attitudes improves group performance.

\section{Human Subject Experiment 3: Collaborative Design with Hyperinterac- tive Evolutionary Computation}

The third experiment aimed to test Hypothesis H3: Diversity of available evolutionary operators improves group performance. This naturally follows the results of the second experiment discussed above. For the third experiment, we used interactive evolutionary computation (IEC) [15] as an experimental platform, and developed a new framework called "Hyperinteractive Evolutionary Computation (HIEC)" that offers users more controllability than conventional IEC. More details of HIEC can be found in [16], and its overview is also provided in Supplemental Materials. This experiment consisted of two parts: (i) directly manipulating the availability of evolutionary operators and (ii) electronically recording their usage.

The first part of the experiment took place in 2008. A total of 21 students were randomly divided into 7 groups. Each group was given 10 minutes to collaboratively design an "interesting" swarming pattern produced using the Swarm Chemistry model [17, 18]. The following four conditions were configured in the HIEC software and assigned randomly to each group:

- Baseline condition: Neither mixing nor mutation operators were available.

- Mixing condition: Only the operator for mixing two patterns was available.

- Mutation condition: Only the operator for mutating a pattern was available.

- Mixing + mutation condition: Both the mixing and mutation operators were available.

The design process was repeated three times (each time group members were randomly shuffled) so that $3 \times 7=21$ final designs were produced. Those final designs were projected to the screen in the classroom in a randomized order, and then the students evaluated how "cool" each swarm was on a 10-point scale individually and independently. As a result, each design received 21 individual rating scores. The peer evaluation was used to quantitatively assess the quality of the final designs made in each condition.

Students' evaluation results were first normalized so that the mean was 0 and the standard deviation 1 for each individual student's responses, in order to equalize the contribution of each student's ratings to the 


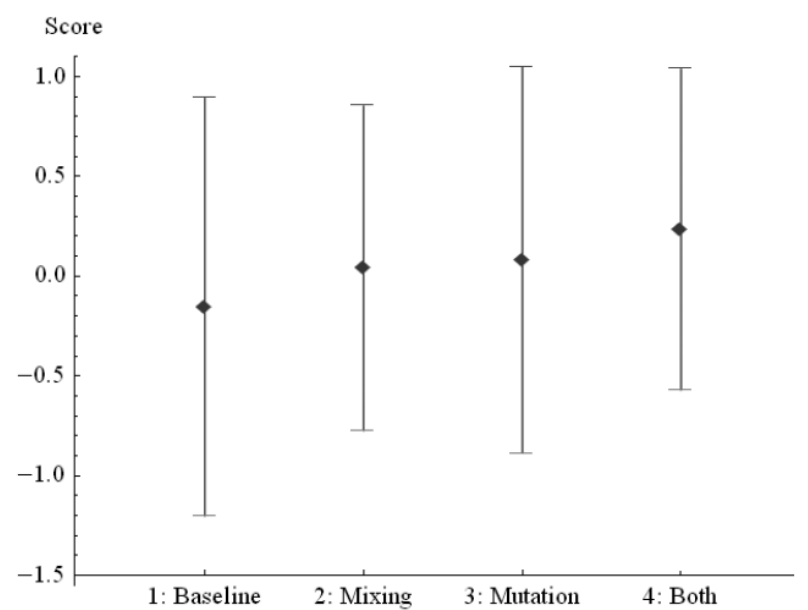

Figure 6: Results of Experiment 3(i). Comparison of average normalized rating scores of final designs produced by groups under different conditions. Mean normalized scores are shown by diamonds, with error bars around them showing standard deviations. A statistically significant difference was detected among the four conditions by ANOVA ( $p=0.0151$ ), while Tukey's and Bonferroni's post-hoc tests detected a significant difference between conditions 1 and 4 .

overall statistics. Then the normalized scores were collected and averaged for each experimental condition. Figure 6 summarizes the results. A statistically significant difference was detected among different conditions. Tukey's and Bonferroni's post-hoc tests detected a significant difference between conditions 1 and 4, supporting Hypothesis H3, i.e., the availability of more evolutionary operators makes the outcomes of group decision making better. It was also observed that each of the Mixing and Mutation operators contributed nearly independently to the improvement of the design quality [18].

The second part of the experiment used a modified version of the HIEC software so that we could electronically record a complete time-stamped log of every single evolutionary event that happened in the same swarm design task. Such a detailed log of decision making processes allowed us to quantitatively analyze how variation and selection occurred in human decision making. This part of the experiment was conducted twice in two separate years (in 2009 and 2010) using the same protocol described below.

Students were randomly divided into groups of three (or sometimes four). Each group was assigned to a station with a touch-screen PC running the modified HIEC software. Each group was given 10 minutes to work on the same collaborative design task as in the first part, with no further guidance given. This phase of the experiment served as the experimental control (Control condition). Then the subjects were reshuffled into new groups. Half of the groups were primed to be critical and risk-averse, with the following written instruction:

Critical condition: Promote and maintain critical attitude throughout the design process. Incremental improvement of existing designs is the key to making a reliable idea. Completely new designs will never be better than well-tested ones. 
The other half of them were primed to be creative and adventurous, with the following written instruction:

Creative condition: Promote and maintain creative attitude throughout the design process. Crazy inspiration and idiosyncratic thinking is the key to breaking the barrier of stereotyped designs. Incremental improvement of existing designs will never work out.

Then the groups were once again given 10 minutes to work on the collaborative design task. Finally, the above step was repeated one more time after another group reshuffling.

A total of 40 students participated in this experiment, forming 39 sessions (= 13 groups $\mathrm{x} 3$ times). Three sessions had technical problems during the experiment and thus their data were excluded from the analysis. As a result, we collected data from 11 sessions working under the control condition, 12 under the creative condition, and 13 under the critical condition. A log file containing detailed information about all the evolutionary events in each session was saved in a local hard drive of each PC, and later collected for post-experimental analysis.

Similarly to Experiment 2, the final designs were evaluated by third parties, i.e., 22 students who took the same course BE-461 in the following year. All the final designs were projected in a randomized order on a screen in the classroom, and then those students rated the quality of each product on a 0-10 scale individually and independently. The average of those rating results was used as the average rating score of each design.

Figure 7 summarizes the results, which were strikingly similar to those of the previous experiment (Fig. 4); the creative groups produced most ideas in this experiment (Fig. 7(a)) while the quality of final designs produced by control groups were comparable to those by creative groups (Fig. 7(b)). It was also observed that creative groups tended to use more mixing operators, critical groups focused more on mutation, and control groups sat somewhere in between (Fig. 7(c)), which supports the observation and interpretation obtained in Experiment 2. These findings clearly show that priming conditions did affect groups' attitudes in discussion, directly relating human behavior in decision making with evolutionary concepts.

Moreover, the use of HIEC software made it possible to collect detailed trajectories of how ideas have been explored and how a final decision has been developed over time. Figure S2 in Supplemental Materials presents visualization of a sample genealogy of ideas generated based on the actual data taken from one of the groups in this experiment. Visual inspection of the idea genealogies indicate that there are some topological variations associated with the priming conditions. More systematic, quantitative analysis of topologies of those genealogies is to be reported elsewhere. 
(a)

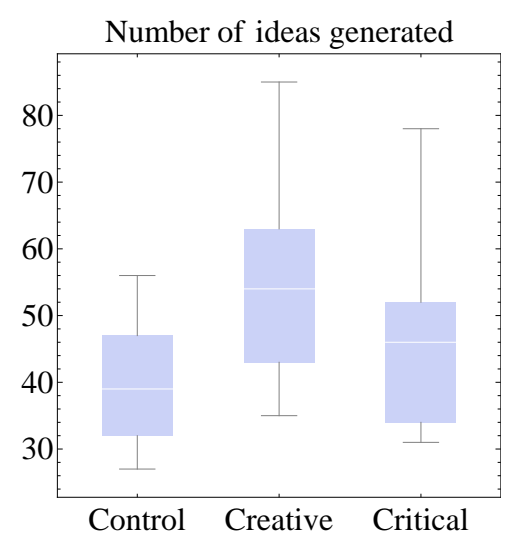

(b)

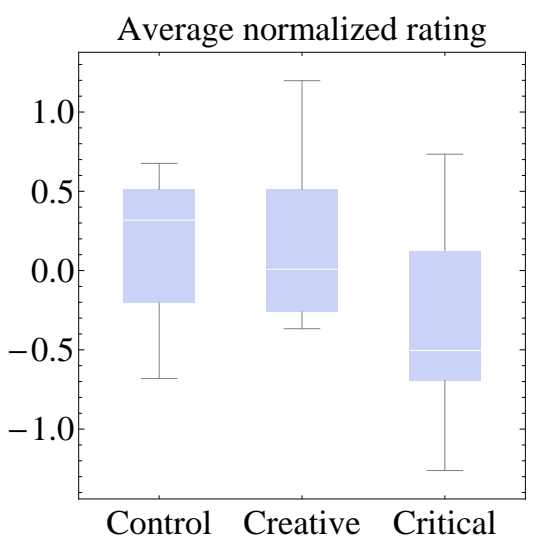

(c)

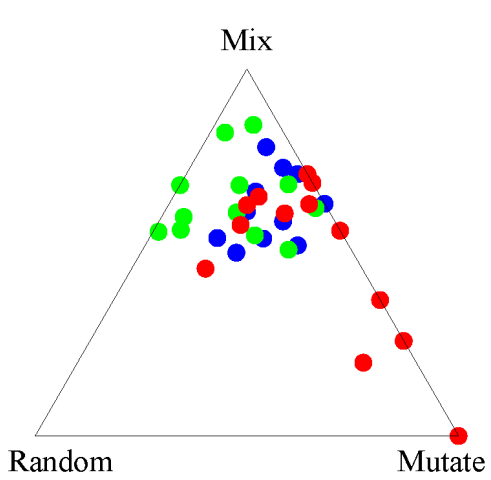

Figure 7: Results of Experiment 3(ii), comparing the distributions of outcomes between three conditions. (a) Number of ideas generated. A moderate difference was detected among the three conditions by ANOVA ( $p=0.0547$ ), while Tukey's posthoc test indicated a significant difference between control and creative conditions. (b) Average normalized rating score of final designs (positive: good, negative: bad). No statistically significant difference was detected by ANOVA $(p=0.0797)$. (c) Ternary plot of the group behavior data with respect to the mix, mutate, and random evolutionary operators. Each marker represents data taken from one group (blue: control, green: creative, red: critical).

\section{Conclusions}

We have presented a series of experiments that illustrate how the dynamics of collective human decision making processes can be studied as evolutionary processes. The results obtained from our human subject experiments nicely matched the predictions made by the computational experiments, illustrating the validity of evolutionary understanding of human decision making and creativity processes. In those experiments, evolutionary computation (EC) played multiple roles, as a theoretical/computational modeling framework as well as an experimental tool for data collection. We believe our work demonstrates untapped potential of EC for interdisciplinary research involving human and social dynamics.

One particularly important take-home message this project has provided us with is the nontrivial role of "diversity" in groups. While the diversity of problem understanding may cause intra-group conflicts and thereby harm the group performance, the diversity of behavior in discussion (e.g., balance between variation and selection) can offer various evolutionary paths in decision making processes that will help improve the group performance. Our results also led us to a conjecture that humans are naturally most balanced in their 
behavior, which could also be explained from an evolutionary viewpoint.

The key findings obtained in this project support our framework that uses evolutionary principles to describe collective human decision making and creativity. This research has presented a conceptual as well as technical shift of focus from human individuals to the ideas evolving through discussions. We hope that this will lead to a theoretical advancement from a traditional, individually-focused psychological or social science paradigm to a more dynamic, multilevel, evolutionary paradigm for collective social processes.

For more information about this project, see the official project website [7].

\section{Acknowledgments}

We thank Francis J. Yammarino, Craig Laramee, David Sloan Wilson, J. David Schaffer, Dene Farrell, Andrew Talia, Benjamin James Bush, Chanyu Hao, Hadassah Head, Thomas Raway, Andra Serban, Alka Gupta and Jeffrey Schmidt for their help in conducting this project. This work was supported in part by the National Science Foundation, under Grants SES-0826711 and DUE-0737313.

\section{References}

[1] H. Sayama and S. D. Dionne, "Using evolutionary computation as models/tools for human decision making and creativity research," in Proc. 4th IEEE Symp. Artif. Life, 2013, pp. 35-42.

[2] P. Bentley, Evolutionary design by computers, Morgan Kaufmann, 1999.

[3] P. J. Bentley and D. W. Corne, Creative evolutionary systems, Morgan Kaufmann, 2002.

[4] M. Klein, H. Sayama, P. Faratin, and Y. Bar-Yam, "The dynamics of collaborative design: Insights from complex systems and negotiation research," Concurrent Engineering: Research and Applications, vol. 11, pp. 201-209, 2003.

[5] N. L. Kerr and R. S. Tindale, "Group performance and decision making," Annual Review of Psychology, vol. 55, pp. 623-655, 2004.

[6] E. Salas, K. C. Stagl, and C. S. Burke, "25 years of team effectiveness in organizations: Research themes and emerging needs," in C. L. Cooper and I. T. Robertson, eds., International Review of Industrial and Organizational Psychology, vol. 19, pp. 47-91, 2004. 
[7] H. Sayama et al., "Evolutionary Perspective on Collective Decision Making project website", http://coco.binghamton.edu/NSF-HSD.html, 2008-2012.

[8] M. Turoff, "Delphi-conferencing: Computer-based conferencing with anonymity," Technological Forecasting and Social Change, vol. 3, pp. 159-204, 1972.

[9] T. Connolly, L. M. Jessup, and J. S. Valacich, "Effects of anonymity and evaluative tone on idea generation in computer-mediated groups," Management Science, vol. 36, pp. 689-703, 1990.

[10] A. R. Dennis, "Information exchange and use in group decision making: You can lead a group to information but you can't make it think," MIS Quarterly, vol. 20, pp. 433-457, 1996.

[11] P. Vitharana and K. Ramamurthy, "Computer-mediated group support, anonymity and the software inspection process: An empirical investigation," IEEE Transactions on Software Engineering, vol. 29, pp. 167-180, 2003.

[12] R. Dawkins, The Selfish Gene. Oxford University Press, 1976.

[13] R. Aunger, ed., Darwinizing Culture: The Status of Memetics as a Science. Oxford University Press, 2001.

[14] D. Farrell, H. Sayama, S. Dionne, F. Yammarino, and D. S. Wilson. "Evolutionary perspective on collective decision making," in Proc. 7th Intl. Conf. Complex Systems (ICCS 2007), 2007.

[15] H. Takagi, "Interactive evolutionary computation: Fusion of the capabilities of EC optimization and human evaluation," Proc. IEEE, vol. 89, no. 9, pp. 1275-1296, 2001.

[16] B. J. Bush and H. Sayama, "Hyperinteractive evolutionary computation," IEEE Trans. Evol. Comput., vol. 15, pp. 424-433, 2011.

[17] H. Sayama, "Swarm chemistry,” Artif. Life, vol. 15, pp. 105-114, 2009.

[18] H. Sayama, S. Dionne, C. Laramee, and D. S. Wilson, "Enhancing the architecture of interactive evolutionary design for exploring heterogeneous particle swarm dynamics: An in-class experiment," in Proc. 2nd IEEE Symp. Artif. Life, 2009, pp. 85-91. 